The feeling of me feeling for you: Interoception, alexithymia and empathy in autism.

Short title: Interoception, alexithymia and empathy in autism.

Cari-lène Mul - Anglia Ruskin University ${ }^{1}$

Steven D. Stagg - Anglia Ruskin University ${ }^{1}$

Bruno Herbelin - Center of Neuroprothetics, EPFL ${ }^{2}$

Jane E. Aspell - Anglia Ruskin University ${ }^{1}$

${ }^{1}$ Department of Psychology

Faculty of Science and Technology

Anglia Ruskin University

Cambridge

CB1 1PT

United Kingdom

${ }^{2}$ EPFL Center of Neuroprosthetics

Campus Biotech Batiment H4

Chemin Des Mines 9

CH-1202 Geneva

Switserland

The authors declare no conflict of interest. 


\begin{abstract}
Following recent evidence for a link between interoception, emotion and empathy, we investigated relationships between these factors in Autism Spectrum Disorder (ASD). 26 adults with ASD and 26 healthy participants completed tasks measuring interoception, alexithymia and empathy. ASD participants with alexithymia demonstrated lower cognitive and affective empathy than ASD participants without alexithymia. ASD participants showed reduced interoceptive sensitivity (IS), and also reduced interoceptive awareness (IA). IA was correlated with empathy and alexithymia, but IS was related to neither. Alexithymia fulfilled a mediating role between IA and empathy. Our findings are suggestive of an alexithymic subgroup in ASD, with distinct interoceptive processing abilities, and have implications for diagnosis and interventions.
\end{abstract}

Keywords: autism, interoception, empathy, alexithymia 


\section{The feeling of me feeling for you: Interoception, alexithymia and empathy in autism}

\section{Introduction}

Autism Spectrum Disorder (ASD) is a developmental disorder that is characterised by social and communication impairments, restrictive and repetitive behaviours (DSM-5, American Psychiatric Association, 2013). One of the main characteristics of ASD is an impairment in reciprocal social behaviour. An important psychological explanation that may partly explain this deficit is that it arises from a limited understanding of others' points of view, thoughts, intentions and beliefs, and is known in the literature as a deficit in 'Theory of Mind' (ToM). The understanding of others' emotions is also affected, with studies showing reduced empathy in ASD (Baron-Cohen, Leslie, \& Frith, 1985; Baron-Cohen \& Wheelwright, 2004). However, in recent years it has been recognised that empathy is a multifaceted process with an affective component and a cognitive component, where a feeling of sharing the emotion of another (affective empathy) may arise from the apprehension or comprehension of another's emotional state (cognitive empathy) (Bird \& Viding, 2014; Eisenberg, 2000) as well as from bottom-up processes that result in the vicarious experience of an emotion observed in another, through mirroring mechanisms (Bird \& Viding, 2014; Keysers \& Gazzola, 2009). Several studies have shown that cognitive empathy, which involves inferring the emotion of others, may be reduced in ASD (e.g. Demurie, de Corel \& Roeyers, 2011; Golan \& BaronCohen, 2006), whereas affective empathy (the ability to share the emotion of others) may not be adversely affected (Dziobek et al., 2008; Rogers, Dziobek, Hassenstab, Wolf, \& Convit, 2007; Rueda, Fernández-Berrocal, \& Baron-Cohen, 2015).

Given growing evidence for important links between the detection of internal bodily states (interoception), emotion processing, and empathy (Craig, 2002; Damasio, 2000; Grynberg \& Pollatos, 2015; Herbert, Pollatos, \& Schandry, 2007; Terasawa, Moriguchi, 
Tochizawa, \& Umeda, 2014), the present study will investigate relationships between these factors in participants with ASD.

Interoception - the sense of the physiological state of the body (Craig, 2002) - has been proposed to play an important role in emotion processing (Craig, 2002; Damasio, 2000; James, 1894), and may also play a part in the process of empathy. There is some evidence that people who are more sensitive to their bodily (interoceptive) sensations tend to have a better understanding of their emotions (Critchley, 2005; Herbert, Herbert, \& Pollatos, 2011), and experience emotions more intensely (Herbert et al., 2007; Wiens, Mezzacappa, \& Katkin, 2000). Interoceptive sensitivity (IS) is usually objectively measured by tasks that assess the accuracy with which one detects one's heartbeat (Cameron, 2001; Schandry, 1981; Whitehead, Drescher, Heiman, \& Blackwell, 1977). Alternatively, subjective interoceptive awareness (sometimes called interoceptive sensibility - see Garfinkel et al., 2015) may be measured with questionnaires (e.g. Mehling et al., 2012; Porgess, 1993). Lastly, metacognitive measures of interoceptive awareness, quantifying the discrepancy between subjective awareness and objective sensitivity, have also been used (Garfinkel et al., 2015). Studies have found a relationship between IS and cognitive and affective empathy for pain (Singer et al., 2004), and a cortical index of interoception - the heartbeat evoked potential has been associated with empathic concern (Fukushima, Terasawa, \& Umeda, 2011). Furthermore, neuroimaging studies show that the primary brain region involved in interoception - the insula - is also activated during the subjective awareness of emotional feelings, including anger, disgust, pain, and empathy (Craig, 2008; Ochsner et al., 2008; Singer, Critchley, \& Preuschoff, 2009; Zaki, Davis, \& Ochsner, 2012), and insula activation during empathy tasks can be increased by enhancing interoceptive awareness beforehand (Ernst, Northoff, Bäker, Seifritz, \& Grimm, 2012). 
These studies suggest that interoceptive brain areas are recruited both during emotional and empathy processes. Moreover, similar brain activity occurs when experiencing emotions and observing others' emotions (Botvinick et al., 2005; Jabbi, Bastiaansen, \& Keysers, 2008; Wicker et al., 2003). Additionally, learned associations between interoceptive signals and emotions observed in others may contribute to empathy (Bird \& Viding, 2014; Quatrocki \& Friston, 2014). Bird and Viding (2014) have proposed the Self to Other Model of Empathy (SOME) in which interoceptive cues and the representation of these in emotions, together with a situation understanding system and ToM, provide the basis for empathy when the experience of emotion is recognised as being elicited by the emotions of others. Having an internal representation of one's own emotions is an integral part of this empathy model.

In this light, it is important to note that ASD is a disorder that co-occurs with alexithymia (Hill, Berthoz, \& Frith, 2004), a condition characterised by a reduced ability to recognise, describe and understand one's own emotions (Nemiah, Freyberger, \& Sifneos, 1976). Alexithymia is considered to be a trait that is prevalent in around $10 \%$ of the general population (Linden, Wen, \& Paulhus, 1995), but prevalent in $\sim 50 \%$ of the ASD population (Hill et al., 2004; Samson, Huber, \& Gross, 2012). Indeed, the high prevalence of alexithymia in the autistic population may explain mixed results in emotion research, as results sometimes differ depending on the proportion of ASD participants in any particular sample who have co-occurring alexithymia (Bird \& Cook, 2013; Cook, Brewer, Shah, \& Bird, 2013; Shah, Catmur \& Bird, 2016; Shah, Hall, Catmur \& Bird, 2016). Differences in the recognition of facial expressions have been related to alexithymia, not autism (Cook et al., 2013; Grynberg et al., 2012), and differences in insula activation during empathy tasks between people with and without ASD were not significant after accounting for alexithymia (Bird et al., 2010; Silani et al., 2008). Given these results, it is very important to take alexithymia levels into account when investigating empathy and other traits in ASD. 
There are early indications that interoceptive processing in people with ASD is altered. Sensory processing differences in ASD are well known and studies have reported relative indifference or hyposensitivity to interoceptive sensations like pain, temperature, hunger, and thirst in both adults and children with ASD (Ben-Sasson et al., 2009; Crane, Goddard, \& Pring, 2009; Elwin, Ek, Kjellin, \& Schröder, 2013), although there are some indications of increased sensitivity to pain (Fan, Chen, Chen, Decety, \& Cheng, 2014).

So far, only five quantitative studies have specifically investigated interoception in ASD, and these have reported mixed results, albeit with an overall trend towards reduced interoception (see review by DuBois, Ameis, Lai, Casanova, \& Desarkar, 2016). Enhanced IS was found in a sample of children with ASD in trials that required them to count their own heartbeats for 100 seconds, while no difference was found in trials of shorter duration (Schauder, Mash, Bryant, \& Cascio, 2014). On the other hand, reduced IS was found in a study with adults with ASD (Garfinkel, Tiley, O'Keeffe, Harrison, Seth, \& Critchley, 2016). However, the latter study also reported higher scores on a self-report measure of awareness of bodily feelings. Interestingly, they found that the magnitude of discrepancy between the selfreport measure and IS was indicative of participants' scores on the Cambridge Empathy Quotient. A third study reported reduced interoceptive awareness (measured by self-report) in adults with ASD (Fiene \& Brownlow, 2015). Finally, two recent studies found that alexithymia, not severity of autistic traits, was associated with reduced IS in a typical sample and two samples that included individuals with ASD (Shah et al., 2016a; Shah et al., 2016b).

Providing a possible explanation for the relationship between interoception and empathy, it has been suggested that the social difficulties of ASD arise from malfunctions in the 'oxytocin-interoception system' (Quattrocki \& Friston, 2014), a system that influences the saliency of interoceptive signals, emotions, and the sense of self, from infancy. However, Brewer and colleagues have convincingly pointed out that alexithymia may be a consequence 
of a compromised 'oxytocin-interoception system', not ASD (Brewer, Happé, Cook \& Bird, 2015). The latter is also in line with Bird and Viding's empathy model, who propose that low empathy in autism is caused by impairments in the ToM and situation understanding parts of the SOME, whilst alexithymia may be the result of impairments in the affective representation system of the SOME (Bird \& Viding, 2014).

The present study aimed to further our knowledge of interoceptive processing in ASD by investigating the relations between interoception, alexithymia and empathy. Given research that shows alexithymia to be an explanatory factor of low levels of empathy in ASD (Bird \& Cook, 2013), and following on from the suggestion that alexithymia may be a consequence of differences in the 'oxytocin-interoception system' (Brewer et al., 2013), and being theoretically supported by Bird and Viding's theoretical model of empathy (Bird \& Viding, 2014), we wanted to test if alexithymia could fulfil a mediating role in the relationship between interoception and empathy. Therefore, our hypothesis was that participants with ASD would have lower interoceptive awareness and IS, contributing to higher levels of alexithymia, and lower empathy. In addition, we predicted that the individuals with ASD who also have alexithymia would have lower levels of empathy than those without alexithymia.

\section{Method}

\section{Participants}

Fifty-two participants (14 female) took part in the study, of which 26 (7 female) had previously received a diagnosis of high functioning autism or Asperger syndrome by a clinician independent from the current study. Participants were recruited via advertising, ASD support groups, autism charities and student populations. They received financial compensation for their time, or course credit for participation. A diagnosis of high 
functioning autism or Asperger's syndrome was accepted for inclusion in the ASD group. Twenty-three participants with ASD attended an Autism Diagnostic Observation Schedule (ADOS) diagnostic interview (Lord et al., 2000) with a psychologist trained in the use of ADOS (one of the authors), to confirm their diagnosis of ASD. Nineteen participants met the diagnostic criteria for autism, and four met the criteria for autism spectrum disorder, according to ADOS guidelines. Written, informed consent was obtained from all participants and the study was approved by the local Faculty's ethics panel.

All participants completed the Autism Quotient (AQ) questionnaire (Baron-Cohen, Wheelwright, Skinner, Martin, \& Clubley, 2001). ASD participants scored higher on the AQ, $M(S D)=31.1(9.3)$, than control participants, $M(S D)=16.7(6.4)$. The participants for whom we were unable to obtain ADOS diagnostic results for scored well above the screening cutoff point of 32 on the AQ. The two groups were matched on sex (7 females in each group), on IQ (ASD group IQ $M(S D)=113.8(12.0)$ and control group IQ $M(S D)=110.9(13.5), t$ $(50)=0.83, p=.41)$ measured with the Wechsler Abbreviated Intelligence Scale, $2^{\text {nd }}$ edition (Wechsler, 2011), and on age (ASD group age $M(S D)=25.9$ (7.3), control group age $M(S D)$ $=25.4(7.6), t(50)=0.15, p=.88)$. Five participants with ASD reported a diagnosis of cooccurring anxiety or mood disorder. None of the members of the control group reported any mental disorders.

\section{Materials}

i. Alexithymia measure

Participants completed the 20-item Toronto Alexithymia Scale (TAS-20; Bagby, Parker, \& Taylor, 1994), which assesses alexithymia with statements such as "It is difficult for me to find the right words for my feelings", and participants indicate their level of agreement on a 5 point scale. The TAS-20 has 3 subscales: Difficulty Describing Feelings, Difficulty 
Identifying Feeling, and Externally-Oriented Thinking. The TAS-20 has good reliability and validity (Parker, Taylor, \& Bagby, 2003).

ii. Empathy measures

Empathy was measured in two ways: by an empathy questionnaire designed to measure affective and cognitive empathy separately, and by a behavioural measure of affective empathy. The Questionnaire of Cognitive and Affective Empathy (QCAE; (Reniers, Corcoran, Drake, Shryane, \& Völlm, 2011) collated 31 items for commonly used and validated empathy questionnaires that reflect cognitive empathy, defined as "the ability to construct a working model of the emotional states of others", and affective empathy, defined as "the ability to be sensitive to and vicariously experience the feelings of others" (Reniers et al., 2011).

The English core version of the Multifaceted Empathy Test (MET; (Dziobek et al., 2008) provided a behavioural measure of affective empathy. This version consists of 40 images displaying an individual in an emotional state in a naturalistic environment, which were presented one by one on a 17" computer screen using E-prime 2.0 software (Psychology Software Tools, Pittsburgh, PA). Participants were asked to infer the emotion the person depicted is experiencing by choosing one of four words that best described the emotion displayed. The second task (measuring affective empathy) was to indicate on a scale of 1 to 9 how much they empathized with the person depicted, with empathizing being defined as feeling the same emotion as the one the person on the screen is showing. The definition of empathizing was given at the start of the presentation of the relevant block of pictures, while the question appeared as a caption above each picture. The pictures were shown in random order in eight blocks of ten, and the blocks alternated between emotion recognition and empathizing. 


\section{iii. Interoception tasks}

We measured the accuracy with which participants perceived their heartbeat; this will be referred to as interoceptive sensitivity (IS). There are two widely used heartbeat perception tasks: One task measures the performance at heartbeat discrimination and the other at heartbeat tracking. At the time the present study was designed, no research into interoception and ASD had been published, and there is still no consensus in the field as to which is a better measure of interoception: both have their advantages and disadvantages and measure interoception in somewhat different ways (Knoll and Hoddap, 1992; Brener \& Ring, 2016). The tracking task requires internal monitoring of heartbeats over short intervals and is confounded by time estimation ability and beliefs about heart rates, whereas the discrimination task requires cross-sensory matching of the timing of internal and external events (for more on this see Discussion). Nevertheless, both tasks are widely regarded as measuring interoceptive ability (Garfinkel, et al., 2015) and so it is not inconceivable that they should be complementary in the present study. We could not predict, however, which of the two measures would be different in participants with autism and so we employed both.

During the heartbeat tracking task (Schandry, 1981) the participant was seated upright, and was asked to count their own heartbeats and indicate how many heartbeats had occurred during a specific short time interval, whilst their heartbeat was recorded by an electrocardiogram (ECG). To this end, participants had three disposable surface electrodes with conductive hydrogel attached to their chest, and cardiac activity was relayed through shielded wires to the Powerlab Data Acquisition unit (AD Instruments, Germany). There were 4 trials of 25, 35, 45 and 55 seconds, presented in random order. Participants were asked not to take their pulse and they did not receive any feedback on their performance.

A second measure of IS - a version of the heartbeat discrimination task (Whitehead et al., 1977) - was also administered. During this task, ECG provided the input for in-house 
developed ExpyVR software (http://lnco.epfl.ch/expyvr), which produced brief auditory tones triggered by the R-wave of the ECG in the synchronous condition, or it produced brief tones at a speed of either $80 \%$ or $120 \%$ of the frequency of the participant's preceding two Rwaves to create the asynchronous condition. Participants were exposed to 20 tones per [synchronous or asynchronous] trial and indicated after each trial whether they thought the tones were in time with their heartbeat or not in time. Four synchronous trials and four asynchronous trials (two faster and two slower than the participant's heartbeat) were administered in random order. The order of the two heartbeat tasks was counterbalanced across participants.

Interoceptive awareness (IA) was measured with the 32-item Multidimensional Assessment of Interoceptive Awareness questionnaire (MAIA - (Mehling et al., 2012), which assesses awareness of bodily sensations as a multi-dimensional construct, including elements of regulation and appraisal (Cameron, 2001). It contains statements like "When I am tense, I notice where the tension is located in my body" and participants indicate how often they experience this on a 5 point scale. The MAIA recognises that certain aspects of bodily awareness may be maladaptive; excessive focus on, worrying about or avoidance of bodily sensations are related to higher levels of anxiety, catastrophizing, and more difficulties with emotion regulation (Mehling et al, 2012). Items measuring worrying, avoidance and excessive focus have been reverse scored so that higher scores reflect mentally healthier levels of body awareness rather than more maladaptive awareness. The MAIA has 8 subscales that reflect different dimensions of body awareness: Noticing, Distracting, Worrying, Attention Regulation, Emotional Awareness, Self-regulation, Body Listening, Trusting.

Please note that what is called interoceptive awareness here, is equivalent to what other authors may call interoceptive sensibility (Garfinkel et al., 2015). These authors reserve 
the term interoceptive awareness for meta-awareness of interoceptive sensitivity (a measure that quantifies how aware people are of their accuracy in detecting their own heartbeat), which was not measured here.

\section{Data Analysis}

Differences between groups were analysed with two-tailed independent $t$-tests (Controls vs. ASD participants) or with one-way Anovas (Controls/ASD non-alexithymia/ASD alexithymia). Spearman correlations were conducted between the relevant variables. The ASD group was split into participants with and without alexithymia, according to the high alexithymia cut-off score of 62 (Bagby, Parker, \& Taylor, 1994), for the analyses investigating the effect of alexithymia on empathy.

A 2 x 2 (empathy type $\times$ ASD status) mixed Anova, controlling for alexithymia as a covariant, and a 2 x 3 (empathy type x ASD-alexithymia status) mixed Anova were used to investigate differences between groups on the QCAE data. Post-hoc two-tailed $t$-tests were conducted, where appropriate, with the alpha-level adjusted to .017.

One participant with ASD was excluded from the IS analyses due to not following instructions. For the heartbeat tracking task an accuracy score was calculated using the formula IS $=\left\{1 /{ }_{4} \Sigma[1-(\mid\right.$ recorded heartbeats - counted heartbeats $\mid /$ recorded heartbeats $\left.)]\right\}$ as in Schandry, 1981. Accuracy scores of the heartbeat discrimination task were calculated as percentage correct.

The MAIA questionnaire has 8 subscales and is not intended to provide a summated score of IA (Mehling et al., 2012). Multidimensional scaling of the MAIA responses were undertaken, using the PROXCAL algorithm in SPSS, in order to reduce the 8 dimensions of the MAIA and increase power. 
Partial correlations were conducted where there was a significant relationship between the interoception measures, autistic traits (AQ scores) and alexithymia to establish the relative relationship of alexithymia and autistic traits with interoception. Where correlations were found between any of the interoception measures and alexithymia, and alexithymia and the empathy measures, a mediation analysis was conducted using PROCESS (Hayes, 2013) in SPSS, using a multiple regression approach. The relevant interoception measure was entered as predictor in a mediation model, alexithymia was entered as the mediator, and empathy as the outcome variable. In a separate analysis, ASD status was entered as a moderating variable in the relationship between interoception and alexithymia. PROCESS uses confidence intervals generated by bootstrapping to produce inferential statistics (see Hayes, 2013 for more information). 95\% confidence intervals that do not include zero indicate that the effect is likely a real effect (different from zero) and significant at the .05 alpha level.

\section{$\underline{\text { Results }}$}

\section{i. Alexithymia}

Only two individuals without ASD reached the high alexithymia cut-off point of 62 (Bagby, Parker, \& Taylor, 1994), whereas 13 individuals from the ASD group (50\%) had scores of 62 or above. ASD participants scored significantly higher on alexithymia $(M=59.5, S D=15.9)$ than control participants $(M=46.2, S D=11.1)$ on the TAS-20 questionnaire, $t(50)=3.51, p$ $=.001, d=0.97$. In further analyses where a distinction was made between ASD participants with alexithymia and ASD participants without alexithymia, the two control participants with alexithymia were excluded from the control group.

Alexithymia scores showed a moderate negative correlation with MET affective empathy scores $(r=-.46, p<.001)$, and a strong negative correlation with QCAE scores $(r=$ $-.58, p<.001$ ). The two subscales of the QCAE (cognitive and affective empathy) both 
correlated negatively with alexithymia: QCAE cognitive $r=-.57, p<.001$ and QCAE affective $r=-.40, p=.003$. See Table 1 .

ii. Empathy measures

For the QCAE, there was a significant between-subjects effect of ASD status while controlling for alexithymia levels, $F(1,49)=16.93, p<.001$, indicating that ASD participants $(M=72.6, S D=16.3)$ scored significantly lower on the total QCAE scores than control participants $(M=89.1, S D=13.3)$. An interaction effect of ASD status and empathy type, $F(1,49)=16.27, p=.008$, indicated a different effect per empathy type depending on ASD status. On cognitive empathy, ASD participants scored significantly lower $(M=43.1$, $S D=12.6)$ than the control participants $(M=58.0, S D=5.8), t(50)=4.35, p<.001, d=$ 1.20 , but the affective empathy scores did not significantly differ between groups (ASD $M=$ 29.5, $S D=5.8$; controls $M=32.1, S D=5.0), t(50)=1.74, p=.09, d=0.48$. See Figure 1 .
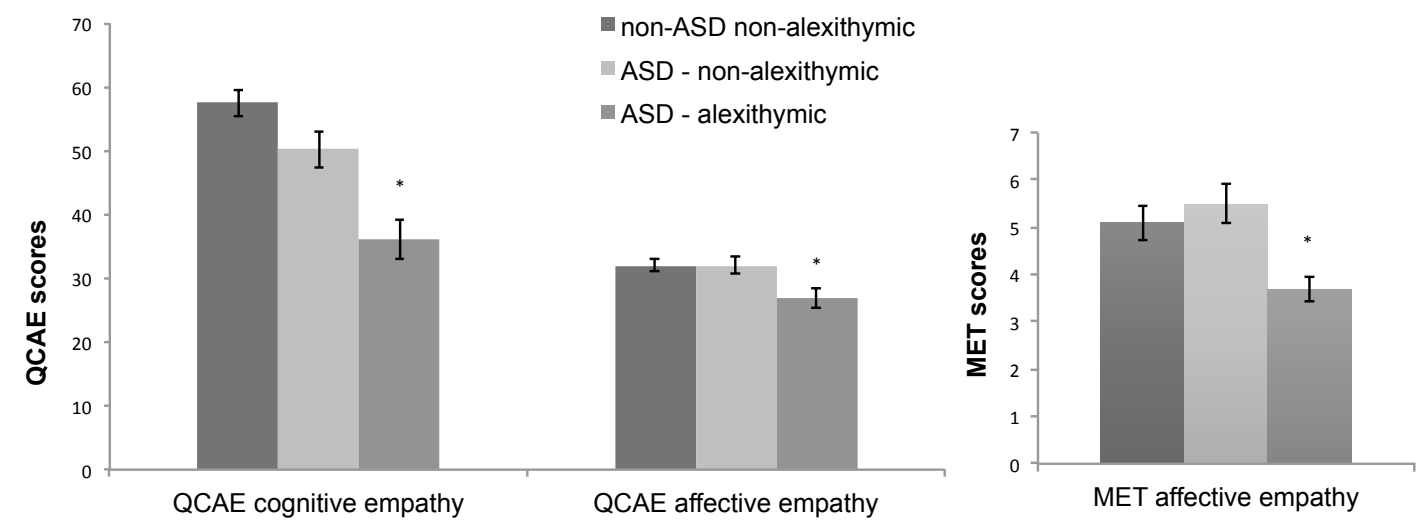

Figure 1. Scores on the empathy measures - the cognitive empathy subscale and the affective empathy subscale of the QCAE and scores on the MET affective empathy task - for control 
participants, ASD participants without alexithymia and ASD participants with alexithymia. Error bars denote standard error. Note $*=p<.05$

A 2 x 3 mixed Anova showed a significant between-subjects effect, $F(2,47)=16.77$, $p<.001$, indicating differences between the control participants, ASD participants without alexithymia and ASD participants with alexithymia. An interaction effect of empathy $\mathrm{x}$ group, $F(2,47)=12.34, p<.001$, indicated that differences between groups were different for the two empathy types. ASD participants with alexithymia had significantly lower affective empathy on the QCAE $(M=26.9 . S D=5.7)$ compared to the control group $(M=$ $32.0, S D=5.1), t(35)=2.78, p=.009, d=0.94$, and lower affective empathy compared to ASD participants without alexithymia, $t(24)=2.48, p=.02, d=0.97$, approaching significance taking into account a Bonferroni corrected alpha-level of 0.017 , although the effect size is large and non-significance may be attributed to the small sample size $(n=26)$. There was no difference in affective empathy between ASD participants without alexithymia $(M=32.1, S D=4.8)$ and the control participants, $t(35)=.02, p=.98, d=0.01$. On cognitive empathy, ASD participants with alexithymia scored significantly lower $(M=36.0, S D=$ 10.97) than control participants $(M=57.5, S D=10.4), t(35)=5.90, p<.001, d=2.00$, and lower than ASD participants without alexithymia $(M=50.2, S D=10.0), t(24)=3.43, p=$ $.002, d=1.34$. ASD participants without alexithymia scored lower on cognitive empathy than the control participants, $t(35)=2.09, p=.04, d=0.71$, approaching the Bonferroni-corrected significance level of .017 . The effect size is medium to large.

There were similar levels of affective empathy - as measured by the MET - in ASD participants and control participants (ASD $M=4.6, S D=1.7$; controls $M=5.0, S D=1.8$ ), $t(50)=0.84, p=.40, d=0.23)$. Using a one-way Anova accounting for the alexithymia status in the ASD group, a significant difference between groups, $F(2,47)=4.05, p=.03$, was found. ASD participants with alexithymia scored significantly lower than ASD 
participants without alexithymia, (ASD $M=3.7, S D=1.5) t(24)=2.97, p=.007, d=1.16$, who scored similar to control participants without alexithymia, (ASD $M=5.5, S D=1.5$, controls $M=5.1, S D=1.8), t(35)=0.66, p=.51, d=0.22$, see Figure 1 .

ASD participants were equally accurate at identifying an emotion displayed on the screen $(M=0.67, S D=0.08)$ as the control participants $(M=0.67, S D=0.1), t(50)=0.12, p$ $=.91, d=0.03$, in the MET emotion recognition task. Alexithymia status of the ASD participants did not alter these results, $F(2,47)=0.17, p=.84$, nor was there a correlation between MET emotion recognition and alexithymia for all participants, $r(50)=.20, p=.15$; and ASD participants separately, $r(24)=.26, p=.20$. Therefore we may assume that the differences seen between groups in the MET affective empathy task were not due to poorer emotion recognition due to alexithymia.

iii. Interoception measures

\section{a. Interoceptive sensitivity}

Statistical checks (independent two-tailed $t$-tests) were made to ensure there were no significant differences in heart rate or Body Mass Index between ASD participants and control participants. Nor were there any significant correlations between heart rate and autistic traits, or BMI and performance on the IS tasks. Only on the heartbeat tracking task was there a trend towards participants with higher BMI to be less accurate at tracking, $r(46)$ $=-.24, p=.07$.

There was no significant difference in the accuracy scores for the heartbeat discrimination task between ASD participants $(\mathrm{M}=0.56, \mathrm{SD}=0.17)$ and control participants, $(\mathrm{M}=0.62, \mathrm{SD}=0.19), t(49)=0.86, p=.39, d=0.24$. Taking into account the alexithymia status of ASD participants in a three level one-way Anova did not alter these results, $F(2,46)$ $=1.92, p=0.16$. However, ASD participants were less accurate $(M=0.63, S D=0.19)$ than control participants $(M=0.74, S D=0.15), t(42.8)=2.10, p=0.04, d=0.58$ on the heartbeat tracking task. See Figure 2. 


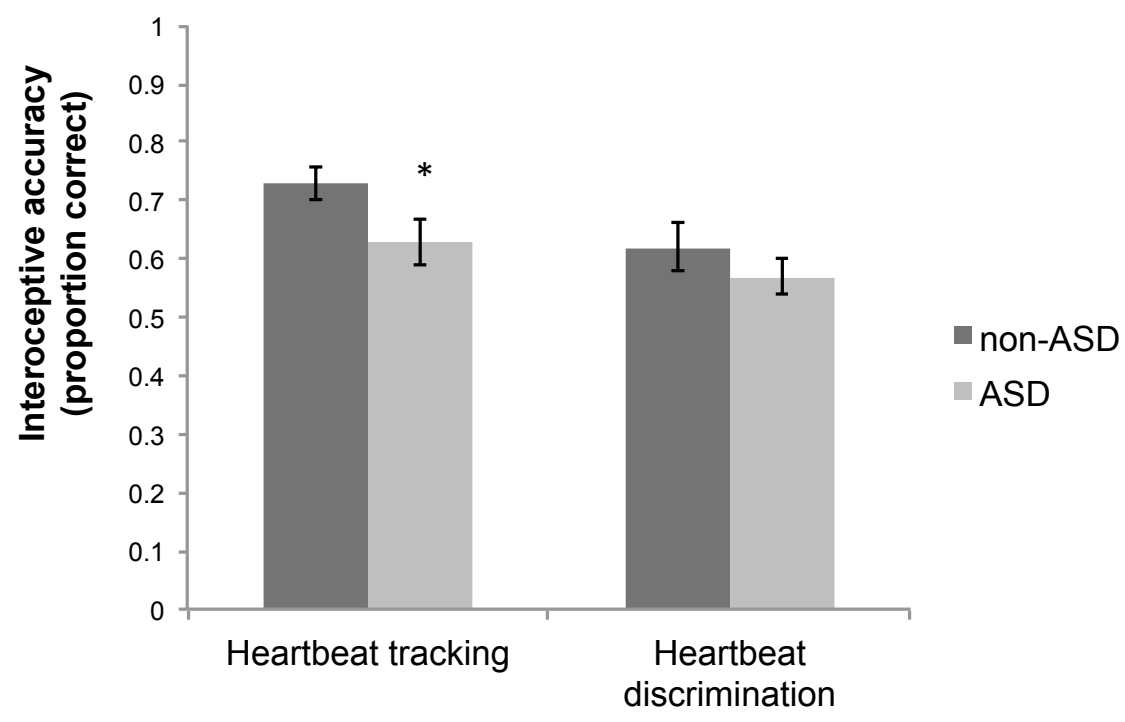

Figure 2. Interoceptive accuracy for control participants and ASD participants, measured by two heartbeat tasks. Error bars denote standard error. Note $*=p<.0$

A three level one-way Anova showed a significant difference between the groups of control participants, ASD participants without alexithymia and ASD participants with alexithymia, $(F(2,46)=3.96, p=.03)$ on heartbeat tracking. Taking into account a Bonferroni-corrected alpha level of .017, the control group had a tendency (approaching significance) to score better than the non-alexithymic ASD group, $t(16.3)=2.69, p=.04$, and the alexithymic ASD group $t(35)=2.08, p=.04$. However, there was no significant difference between ASD participants with alexithymia $(M=0.66, S D=0.13)$ and those without alexithymia $(M=0.59, S D=0.23), t(19.2)=.07, p=0.46)$ on the heartbeat tracking task.

The scores of the heartbeat discrimination task and the heartbeat tracking task did not correlate, $r(49)=0.10, p=.49$. A strong correlation between the two is often cited (Knoll \& 
Hodapp, 1992), but is not always found in studies (e.g. Schulz, Lass-Hennemann, Sütterlin, Schächinger, \& Vögele, 2013). Sometimes a strong correlation is found only in the best performing participants (Schaefer, Egloff, \& Witthüft, 2012). Indeed, this was the case here. The performances in both heartbeat tasks correlated strongly only for those with good heartbeat discrimination scores (based on a median split), $r(9)=.70, p=.02$. There were no significant correlations between IS measures and empathy measures or alexithymia; see Table 1.

\section{b. Interoceptive awareness}

Multidimensional scaling analyses showed three clusters of MAIA dimensions could be identified and named, (normalised raw stress $=0.035)$ :

1. Awareness (noticing and meta-awareness of bodily sensations), which includes the MAIA dimensions of trusting, emotional awareness and noticing.

2. Active and reactive strategies with regard to bodily sensations, which includes the dimensions of not-distracting, not-worrying, self-regulation and body listening

3. Attention regulation, which is the ability to pay attention to the body in an environment of competing stimuli.

ASD participants scored significantly lower on MAIA awareness, $t(50)=2.50, p=.02, d$ $=0.70$, and MAIA active and reactive strategies, $t(50)=2.37, p=.02, d=0.65$, (awareness $M=8.01, S D=2.83$; active reactive strategies $M=8.15, S D=2.31$ ) than control participants (awareness $M=9.79, S D=2.18$; active and reactive strategies $M=9.64, S D=2.22$ ), but there was no significant difference on MAIA attention regulation, $(\mathrm{ASD} M=2.46, S D=$ 1.90; control $M=3.59, S D=2.84) t(50)=1.70, p=.10, d=0.46$, see Figure 3 .

A three level one-way Anova comparing means between control participants, ASD participants without alexithymia and ASD participants with alexithymia showed a significant 
difference between groups on MAIA awareness, $F(2,47)=7.91, p=.001$; on MAIA Active and Reactive Strategies, $F(2,47)=9.58, p<.001$; and a difference approaching significance on MAIA attention regulation, $F(2,47)=3.12, p=.053$. Participants with ASD and alexithymia scored significantly lower on MAIA awareness, $M=6.66, S D=2.94$, than ASD participants without alexithymia, $M=9.36, S D=2.03, t(24)=2.72, p=.01$ and control participants, $M=9.85, S D=2.22, t(35)=3.71, p=.001$. Similarly, participants with ASD and alexithymia scored significantly lower on MAIA Active and Reactive Strategies, $M=$ $6.76, S D=1.97$, than ASD participants without alexithymia, $M=9.53, S D=1.75, t(24)=$ $3.81, p=.001$ and control participants, $M=9.76, S D=2.27, t(35)=4.00, p<.001$. On MAIA attention regulation ASD participants with alexithymia also had lower scores, $M=$ $1.64, S D=0.71$, than ASD participants without alexithymia, $M=3.27, S D=2.36$, and control participants, $M=3.68, S D=2.94$ ), approaching significance after a Bonferroni correction of the alpha level to $.017, t(35)=2.45, p=.019$. There were no significant differences between control participants and ASD participants without alexithymia.

After Bonferroni correction of the alpha level to $p=.0014$, MAIA awareness had strong negative correlations with autistic traits (AQ) and alexithymia (TAS20), strong positive correlations with QCAE total scores and QCAE cognitive empathy scores, and a moderate positive correlation with MET empathizing scores. There was a moderate negative correlation of MAIA active and reactive strategies with autistic traits and a strong negative correlation with alexithymia scores; see Table 1.

In order to identify the relative magnitude of the relationship of alexithymia and autistic traits with IA, partial correlations were carried out. After Bonferroni correction of the alpha level to $p=.025$, these showed that the relationship between MAIA awareness and AQ was no longer significant after controlling for alexithymia, $r=-0.28, p=.03$, as was the relationship of MAIA active and reactive strategies and AQ, $r=-0.10, p=.49$. However, the 
relationship between MAIA awareness and alexithymia was no longer significant either after controlling for AQ, $r=-0.28, p=.05$. On the other hand, the relationship between MAIA active and reactive strategies and alexithymia was still significant after controlling for AQ, $r$ $=-.38, p=.005$.

Table 1.

Correlations between interoceptive measures and autistic traits, alexithymia and empathy measures.

\begin{tabular}{|c|c|c|c|c|c|c|c|}
\hline Measure & $\mathrm{AQ}$ & TAS20 & $\begin{array}{c}\text { MET } \\
\text { accuracy }\end{array}$ & $\begin{array}{c}\text { MET } \\
\text { empathizing }\end{array}$ & $\begin{array}{l}\text { QCAE } \\
\text { total }\end{array}$ & $\begin{array}{c}\text { QCAE } \\
\text { cognitive } \\
\text { empathy }\end{array}$ & $\begin{array}{l}\text { QCAE } \\
\text { affective } \\
\text { empathy }\end{array}$ \\
\hline $\begin{array}{l}\text { Heartbeat } \\
\text { tracking }\end{array}$ & -0.04 & 0.14 & 0.04 & -0.14 & 0.03 & 0.15 & -0.17 \\
\hline $\begin{array}{l}\text { Heartbeat } \\
\text { detection }\end{array}$ & $-0.29 *$ & -0.10 & -0.17 & 0.11 & 0.16 & 0.13 & 0.17 \\
\hline $\begin{array}{l}\text { MAIA } \\
\text { awareness }\end{array}$ & $-0.57 * * *$ & $-0.57 * * *$ & 0.01 & $0.45^{* * *}$ & $0.56 * * *$ & $0.56 * * *$ & $0.33^{*}$ \\
\hline $\begin{array}{l}\text { MAIA } \\
\text { active and } \\
\text { reactive } \\
\text { strategies }\end{array}$ & $-0.44 * * *$ & $-0.57 * * *$ & -0.26 & 0.23 & $0.27 *$ & $0.33^{*}$ & 0.03 \\
\hline $\begin{array}{l}\text { MAIA } \\
\text { attention } \\
\text { regulation }\end{array}$ & $-0.34 *$ & $-0.36^{* *}$ & -0.09 & -0.09 & 0.22 & $-0.31 *$ & 0.10 \\
\hline
\end{tabular}

${ }^{1}$ Correlations were controlled for Body Mass Index

Note: $* p \leq .05$. **p $\leq .01$. ** ${ }^{* * *} p<.001$. 


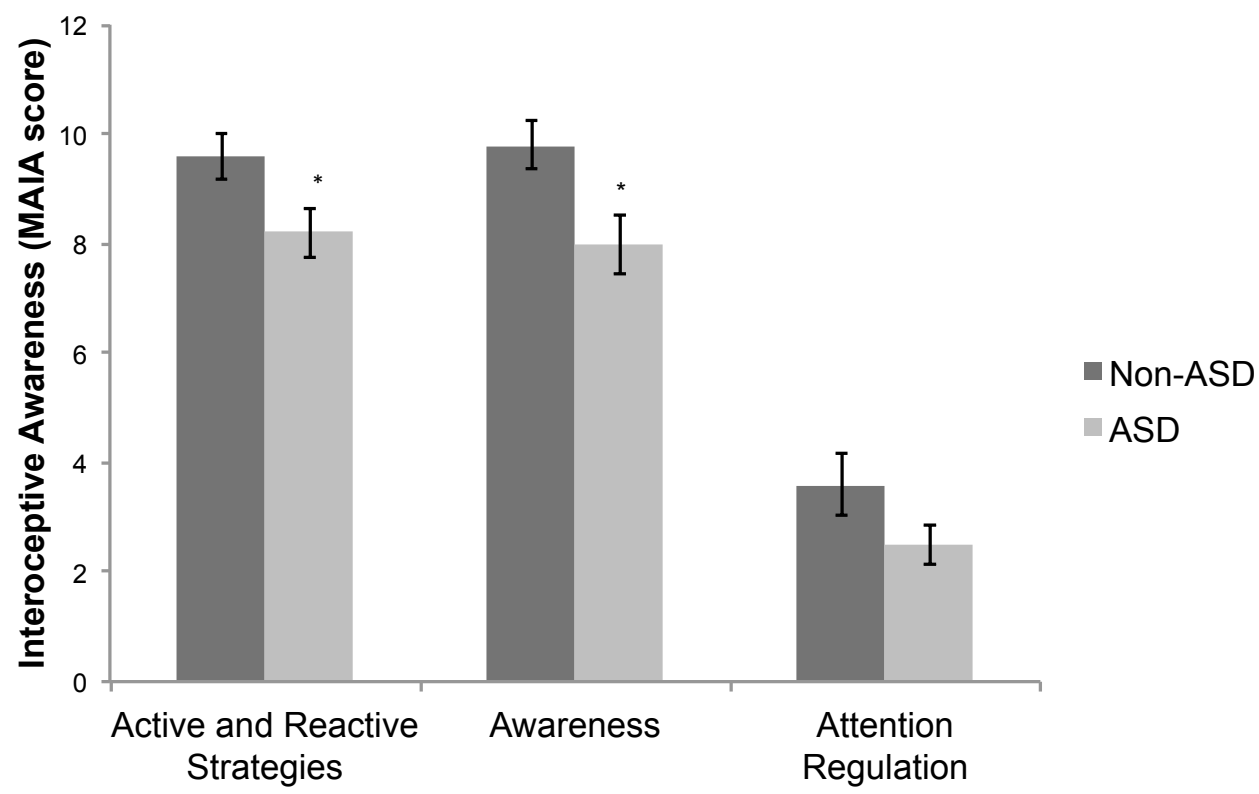

Figure 3. Interoceptive awareness measured by the three clusters of items of the MAIA questionnaire, for control participants and ASD participants. Error bars denote standard error. Note $*=p<.05$

iv. Relationships between interoception, alexithymia and empathy

No conditional process analyses or mediation analyses were conducted with IS as a predictor variable, due to the absence of significant correlations of IS with alexithymia and empathy. Mediation analysis using PROCESS (Hayes, 2013) in SPSS was conducted with the three MAIA clusters entered as predictor variables (using a multiple regression approach), alexithymia as a mediator and total QCAE score as the dependent variable. The analysis showed that both MAIA awareness and MAIA active and reactive strategies contributed significantly to alexithymia, while MAIA attention regulation did not contribute to alexithymia after taking the other MAIA clusters into account (see the regression coeffients $a_{1}, a_{2}$, and $a_{3}$ in Table 2). 
Table 2.

Coefficients of the direct and indirect path of the effect of self-reported interoceptive awareness (MAIA clusters) on self-reported empathy (QCAE) through alexithymia.

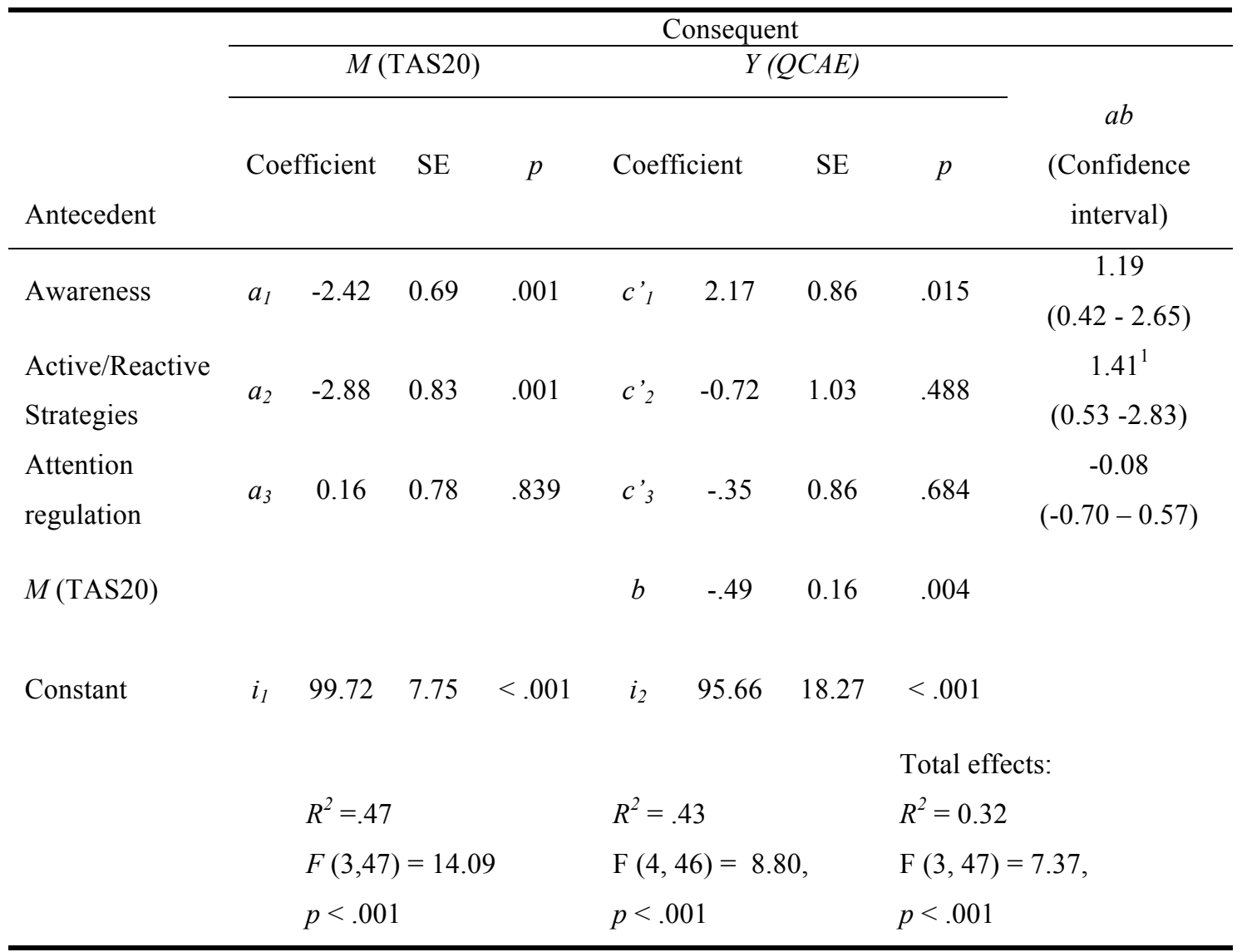

Table 3.

Coefficients of the direct and indirect path of the effect of self-reported interoceptive awareness (MAIA clusters) on behavioural affective empathy (MET) through alexithymia.

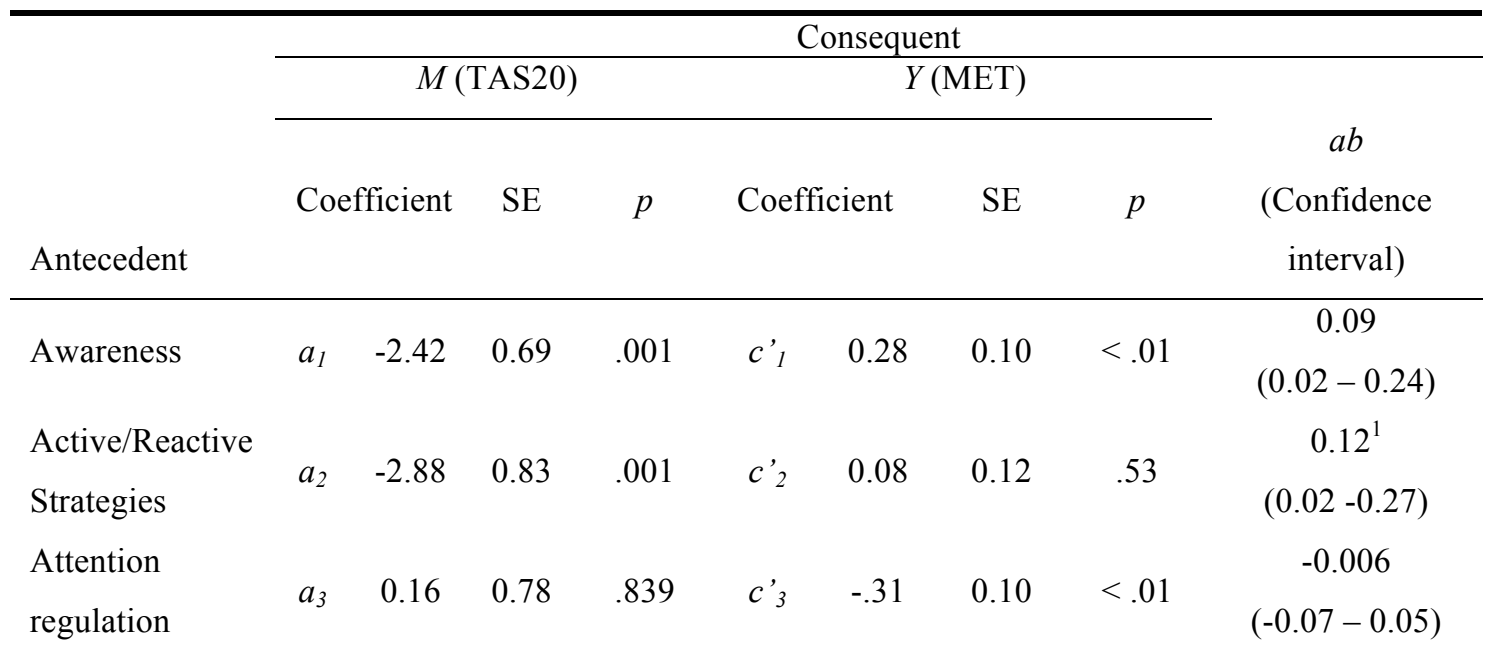




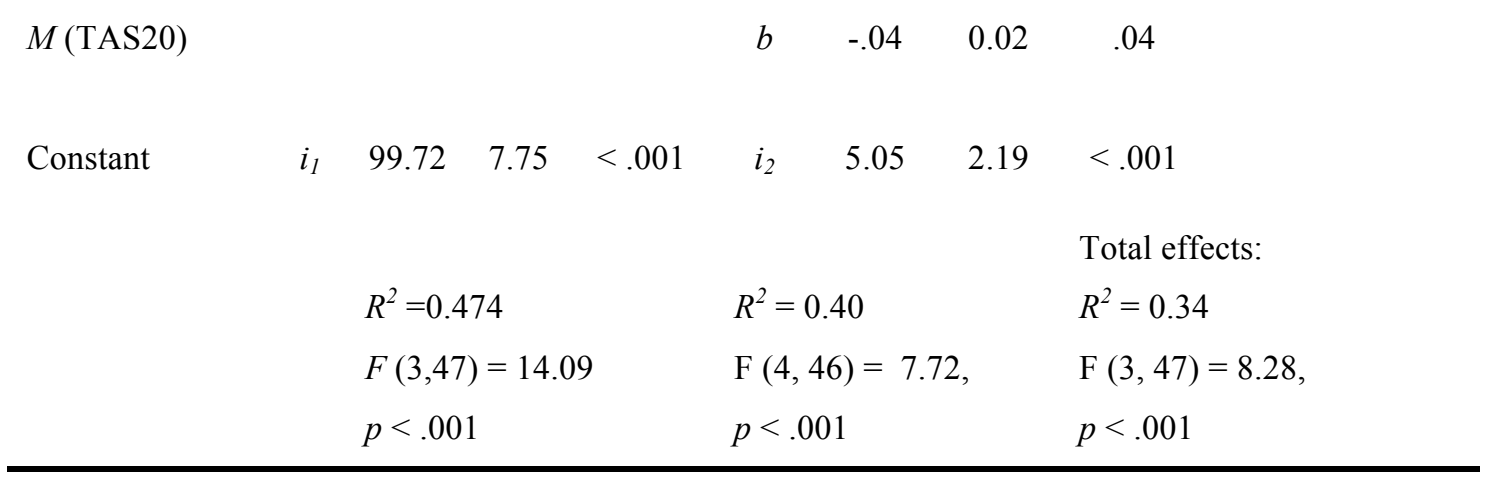

Alexithymia contributed significantly to QCAE scores (see regression coefficient $b$ in Table 2). This indicates that participants with lower MAIA awareness demonstrated higher alexithymia levels (negative coefficients $a_{1}$ and $a_{2}$ ), and in turn participants with higher alexithymia levels tended to have lower QCAE (negative coefficient $b$ ). The indirect path $(a b)$ of the effects of MAIA awareness and MAIA active and reactive strategies via alexithymia on QCAE scores was significant. The three MAIA clusters explained 32 percent of the variance in QCAE empathy scores. See Table 3 for the coefficients of the paths.

The same analysis was conducted to look at the effects of the MAIA clusters on behavioural affective empathy scores (MET empathy) through alexithymia; see Table 3. [Insert Table 3] Similar to the effects of MAIA clusters on QCAE scores, this showed that the indirect effect of MAIA awareness through alexithymia to MET empathy was significant, as was the indirect effect of MAIA Active/Reactive strategies. The effect of MAIA attention regulation on MET empathy was not mediated through alexithymia after taking the other MAIA clusters into account. The three MAIA clusters explained 34 percent of the variance in MET empathy scores.

To determine whether the path from interoceptive awareness to understanding oneself to understanding others differed in participants with ASD, a conditional process analysis was carried out (Hayes, 2013). ASD status was introduced as a moderator of the 
indirect path (see Figure 4), testing the hypothesis that the strength of the indirect effect of MAIA scores on empathy through alexithymia depends on whether one has ASD or not. Only the indirect effect of MAIA Active and reactive strategies on QCAE and MET empathy scores via alexithymia were moderated by ASD status (index of moderated mediation QCAE $=2.08, \mathrm{CI}=0.275-4.558$; index of moderated mediation MET empathy $=0.177, \mathrm{CI}=.028-$ 0.408). This means that the increase in alexithymia due to higher active and reactive strategies scores was significantly larger for ASD participants compared to control participants, $\left(\Delta \mathrm{R}^{2}=.05, F(1,47)=4.03, p=.05\right)$. Therefore, the final model of the relationship between the clusters of the MAIA, ASD, alexithymia and empathy can be visualised as in Fig. 4.

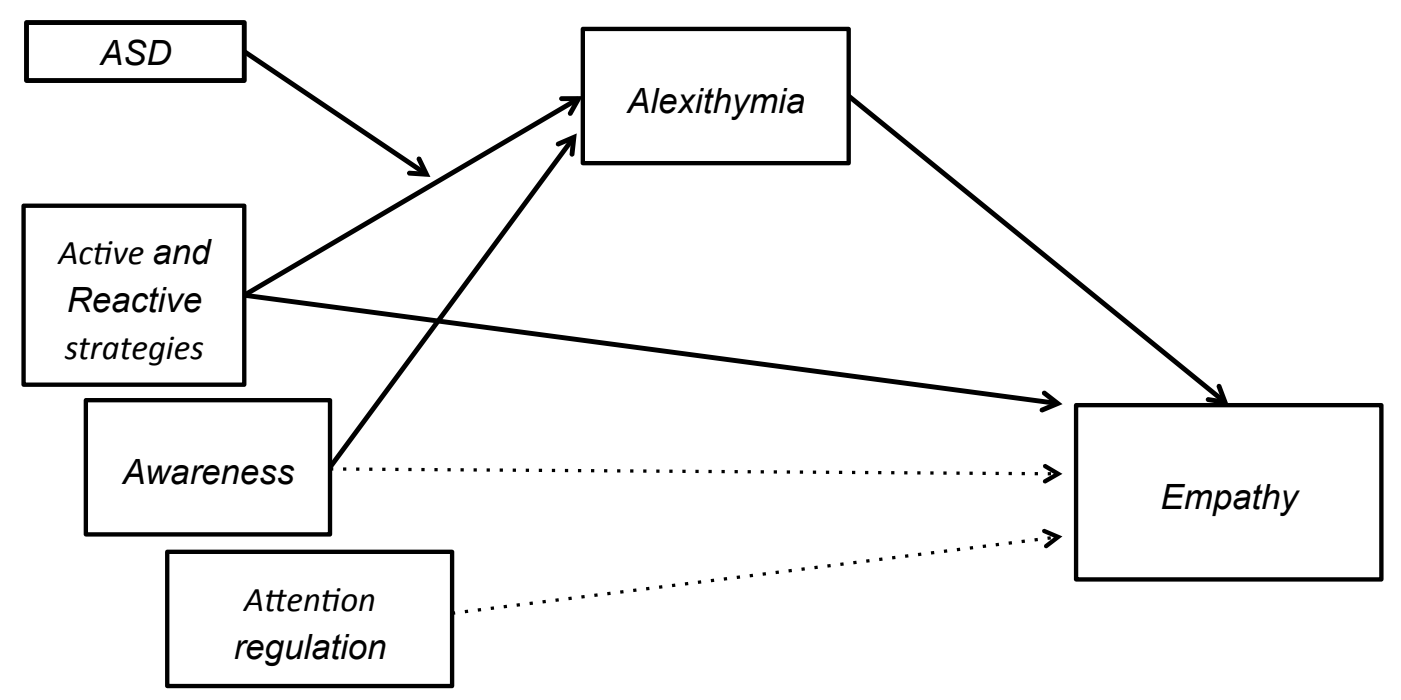

Figure 4. Final mediation model of the relationships between interoceptive awareness (three MAIA clusters), alexithymia (mediator), and empathy. It shows how interoceptive awareness is related to empathy indirectly, via alexithymia, and how ASD status may act as a moderator 
on the indirect relationship. Dotted lines indicate non-significance of an association after taking the other relationships into account with a multiple regression approach.

\section{Discussion}

The present study sought to investigate links between interoceptive processing, alexithymia and empathy in participants with ASD. The findings suggest that interoceptive processing is attenuated in ASD participants compared to control participants, since the former were less accurate at tracking their own heartbeat and showed lower self-reported interoceptive awareness. Contrary to expectations, interoceptive sensitivity (IS) was not related to alexithymia and empathy. However, interoceptive awareness (IA) was related to autistic traits, alexithymia and empathy. IA contributed to empathy levels indirectly, through inverse relationships with alexithymia, suggesting that the greater the awareness of one's own bodily sensations (measured via the MAIA questionnaire), the better one may be able to identify and describe one's own emotions (measured via TAS-20 score), the higher the empathy for others' emotions. It appears then, that the subjective awareness and understanding of one's bodily sensations and emotions is more important for empathy than is accuracy in detecting bodily sensations. These findings can be argued to support Bird \& Viding's SOME (2014), which proposes that cognitive representations of one's own emotions are integral to empathy. The ability to accurately recognise another's emotion, often regarded as a measure of cognitive empathy (Baron-Cohen \& Wheelwright, 2004; Demurie, De Corel, \& Roeyers, 2011; Golan \& Baron-Cohen, 2006), was not related to IS, IA or alexithymia in this sample.

A difference in IS was shown between the groups in one of the two heartbeat perception tasks. On average, control participants were better at tracking their own heartbeat, but there was no difference between groups in the heartbeat discrimination task. The heartbeat discrimination task is thought to be less susceptible to confounds, such as prior 
knowledge about heart rate, but a disadvantage of this task is that most individuals (including current participants) perform close to chance level resulting in a lower variance in performance across a sample (Knapp-Kline \& Kline, 2005; Phillips, Jones, Rieger, \& Snell, 1999). The heartbeat discrimination task relies on a complex integration of stimuli from interoceptive (cardiac) and exteroceptive (auditory) modalities, and requires a temporal comparison of them to enable a judgment of synchronicity. In contrast, the heartbeat tracking task demands attention to only one (interoceptive) modality, and no synchronicity judgement is required. The different demands of these tasks on sensory processing and attention may contribute to the different results for the two tasks in the present study. A dissociation between performance on the two tasks has been found before, with different populations (Kandasamy et al., 2016; Schulz et al., 2013). Performance on the heartbeat tracking task has shown to be related to performance on a time estimation task (Meissner \& Wittmann, 2011), suggesting that the ability to estimate time or sustain attention may influence heartbeat tracking accuracy. However, subsequent research with ASD participants did not replicate this relationship (Shah, Catmur \& Bird, 2016; Shah, Hall, Catmur \& Bird, 2016), nor did controlling for it alter the observed relationship between heartbeat tracking and alexithymia. We did not measure time estimation and could therefore not control for it, but based on the research of Shah and colleagues it appears that time estimation is not always a confounding variable.

Only four studies to date have used heartbeat perception tasks to assess IS in ASD samples. Schauder et al. (2015) found that children with ASD were better at heartbeat tracking than controls, but only in their longest interval (100 secs), which was longer than the longest interval in the present study (55 secs). The authors suggest that the ability to sustain attention to interoceptive signals for longer durations may be better in children with ASD. 
The second study - by Garfinkel et al. (2016) - found, in contrast, reduced IS (measured by the heartbeat tracking task) in 20 adults with ASD, compared to control participants. Our findings are in line with this study, and are also in in line with qualitative reports of sensitivity to interoceptive stimuli in individuals with ASD (Elwin et al., 2013). We note that Garfinkel et al. found enhanced IA (what they term 'interoceptive sensibility'), whereas we found reduced IA. This is likely due to the very different self-report questionnaires used to measure IA: Garfinkel et al. used the Porges Body Perception Questionnaire (Porges, 1993). The items of the Porges questionnaire relate to an interoceptive awareness (or 'sensibility') that is more associated with anxiety, hypochondria and hypervigilance than are the items of the MAIA, for which more positive scores indicate a more 'mindful' attention style towards interoceptive cues, viewed as healthy and beneficial (Mehling, 2016). Given these important differences it is not a contradiction between the studies, and indeed, it could well be expected, given associations between ASD and anxiety (Skokauskas \& Gallagher, 2010), that participants with ASD should score high on the Porges questionnaire, and low on the MAIA.

The third recent study, by Shah et al. (2016b), found no difference in performance on the heartbeat tracking task between 19 adults with ASD and a control group. However, importantly, the two groups were matched for alexithymia, and the authors report that across two experiments IS was highly correlated with alexithymia, but not with autistic traits (AQ scores), leading to their conclusion that alexithymia, and not autism, is associated with reduced IS. This result was replicated in their other study (Shah, et al., 2016a). In contrast, while we did not find a correlation between alexithymia and IS, we did find correlations between alexithymia and interoceptive awareness. The difference with respect to IS may reflect differences in characteristics of the population samples: in the study by Shah et al. there was a greater number of participants with alexithymia than in our sample. Our null- 
result in the relationship between alexithymia and IS should be taken with some caution, because our control group and ASD group were not matched for alexithymia severity, and the variance in alexithymia of the ASD group and the control group was not equal. Despite this, we did not find a significant difference between ASD participants with alexithymia and those without alexithymia on the heartbeat tracking task.

Our data show no correlation between IS and the empathy measures. In contrast, Grynberg and Pollatos (2015) found that IS, measured by the heartbeat tracking task, was predictive of the level of self-reported arousal, compassion and estimated intensity of pain, in response to viewing pictures of others in painful and non-painful situations. As such, empathy was measured as empathy for pain only, which may elicit stronger feelings in the empathic observer than our stimuli. A recent study on healthy adults that measured heartbeat tracking and empathy (via the QCAE) also found no correlation between the two (Ainley, Maister, \& Tsakiris, 2015). Garfinkel and colleagues found that a calculation of metaawareness of IS, by quantifying the discrepancy between IS and self-report IA, was related to scores on an empathy questionnaire (Garfinkel et al., 2016).

In line with Fiene and Brownlow (2015), we found that self-reported interoceptive awareness (measured with the MAIA) was lower for ASD participants, in two of the three clusters (comprising 7 of the 8 subscales of the MAIA): the cluster that measured noticing and meta-awareness of bodily sensations, and the cluster that measured active and reactive strategies towards bodily sensations, which encompasses regulatory aspects of bodily feelings and the tendencies to not worry about bodily feelings. There was no difference between groups for MAIA attention regulation.

When taking into account the alexithymia status of the participants, this showed that ASD participants with alexithymia had lower MAIA scores than those without alexithymia in two of the three MAIA clusters. This supports the recent findings by Shah and colleagues 
(2016a; 2016b) that alexithymia and interoception are related, whereas interoception and autistic traits may not be. The partial correlations we performed to tease out the relative relationships of autistic traits and alexithymia with interoception confirmed this interpretation in the case of MAIA active and reactive strategies; after controlling for alexithymia, the relationship with autistic traits was no longer significant, while the relationship with alexithymia remained significant even after controlling for autistic traits. We could not confirm this for MAIA awareness however, where AQ remains significantly related to MAIA awareness after controlling for alexithymia, and vice versa. Whether this is due to our participant groups not being matched for alexithymia is hard to establish, but cannot be ruled out. Having matched groups on alexithymia would allow for a better assessment of whether the effects of alexithymia shown in the ASD population are also seen in the typical population.

None of the MAIA clusters correlated significantly with the IS measures. This confirms the growing consensus that the conscious experience of bodily sensations does not necessarily reflect one's sensitivity to them (Cameron, 2001; Garfinkel, Seth, Barrett, Suzuki, \& Critchley, 2015). This corroborates previous studies (Garfinkel, et al., 2016; Khalsa et al., 2008). These studies, as well as the present one, therefore reinforce the notion that when investigating interoceptive processes, distinctions should be made between the physiological states of the body, the brain's sensitivity to these states, metacognition of one's sensitivity, and general 'trait' interoceptive awareness (Garfinkel et al., 2015).

The finding that ASD participants have overall lower empathy levels than participants without ASD supports previous findings (e.g., Baron-Cohen \& Wheelwright, 2004; Greimel et al., 2010). When cognitive and affective empathy were separately examined, only cognitive empathy was found to be lower in ASD participants; affective empathy was at the same level as found for control participants, as in other studies (Blair, 2005; Dziobek et al., 
2008; Rogers et al., 2007; Rueda et al., 2015). This result was corroborated by data from the affective empathy behavioural task: there was no difference between ASD participants and controls when they were asked how much they felt the same emotion as the people shown on the monitor. This shows that the description of ASD as an empathy disorder (Gillberg, 1992) is not entirely correct, and difficulties in emotional and social functioning are more likely to reflect a deficit in the cognitive domain rather than the affective domain (Bachevalier \& Loveland, 2006).

However, our results that ASD participants were no worse than control participants on the MET emotion recognition task is not in line with previous research employing this task (Dziobek et al., 2008), and other emotion recognition tasks. Examining the results more closely, this appears to be due to our control participants underperforming, rather than our ASD participants performing better than in Dziobek's study. This may be due to ceiling effects being lower in the English version compared to the German version, the inclusion of a few non-native English speakers, or simply because of our particular sample of participants.

Previous research has suggested that inconsistent findings with regard to empathy levels in participants with ASD might be explained by variations in alexithymia levels among participants (Bird et al., 2010; Cook et al., 2013; Silani et al., 2008). Confirming this, we found that both cognitive and affective empathy levels of the ASD participants with cooccurring alexithymia were lower than those of control participants, whereas in ASD participants without alexithymia only cognitive empathy was affected; they had similar levels of affective empathy to control participants, on both behavioural and self-reported measures. Given the high prevalence of alexithymia amongst ASD participants (here 50\% - in line with earlier findings, e.g. Hill et al., 2004), there may be large subset of individuals with ASD whose experience of emotions (their own and those of others) is qualitatively different from other individuals with ASD. Furthermore, our results suggest that these individuals may have 
reduced access to associative learning mechanisms between interoceptive signals, their emotions, and others' emotions (Bird \& Viding, 2014; Murphy, Brewer, Catmur \& Bird, 2017; Quatrocki \& Friston, 2014), which means interoceptive training to accompany social and/or emotional learning for these individuals could be helpful (Livingston \& Livingston, 201; Shah, 2016). This may be a valuable insight for anyone who deals with people with ASD, from parents to teachers to therapists, and is a topic that clearly warrants further research.

Conditional process analyses showed that alexithymia played a mediating role between IA and empathy, and that this relationship was partly moderated by autistic status. The indirect path, i.e. the association of IA with empathy via alexithymia, explained around a third of the variance in empathy scores. Specifically, lower scores on the MAIA clusters of awareness, and active and reactive strategies, were both associated with higher alexithymia (TAS-20) scores. Higher levels of alexithymia were in turn related to lower levels of empathy, in keeping with earlier findings (Grynberg et al., 2010; Guttman \& Laporte, 2002). ASD status did not moderate the relationship between the cluster of 'awareness' and alexithymia, but it did moderate the relationship between the cluster of 'active and reactive strategies' and alexithymia: If a participant had ASD, the relationship between 'active and reactive strategies' and alexithymia was significantly stronger than if a participant did not have ASD. This may mean that the attitudinal and regulatory dimensions of IA may be more consequential for individuals with ASD than for individuals without ASD. Resulting higher levels of alexithymia and lower levels of empathy may be related to more difficulties in social functioning and emotion regulation (Garfinkel, et al., 2016), and may affect other aspects of self-referenced cognition (Cameron, 2001; Northoff et al., 2006) and mental health (Khalsa \& Lapidus, 2016; Murphy et al., 2017). For example, excessive focus on bodily sensations and a numbing of bodily sensations have been related to maladaptive self- 
referenced processing contributing to anxiety and depression respectively (Dunn et al., 2010; Paulus \& Stein, 2010). Both disorders are also associated with altered alexithymia levels (Marchesi, Brusamonti, \& Maggini, 2000; Paulus \& Stein, 2010) and are common cooccurring conditions of ASD (Skokauskas \& Gallagher, 2010).

However, some caution must be exercised with this possible interpretation for two reasons. The difference in the mediating effect of alexithymia between our ASD participants and control participants may be caused by the lack of variance in alexithymia in the control group; the number of control participants with high alexithymia levels did not match the number of ASD participants with high alexithymia. The observed moderation effect also seems to be in contradiction to result of the partial correlations that show that the relationship between MAIA active and reactive strategies and alexithymia to be robust when controlling for autistic traits, while the relationship between autistic traits and MAIA active and reactive strategies became non-significant when controlling for alexithymia. Similarly, two recent studies in which alexithymia levels between groups was matched, found that severity of autistic traits was not related to IS, whereas alexithymia was related to IS (Shah et al., 2016a; Shah et al., 2016b). Secondly, five of the ASD participants reported co-occurring mood disorders (depression or anxiety), three of which had high alexithymia. We can't exclude this as a second possible explanation for the different effect in the ASD group, as we did not measure levels of anxiety or depression. An inability to control for levels of anxiety or depression is a limitation of the present study.

In conclusion, this study has found evidence for the role of interoceptive awareness in empathy, such that higher interoceptive awareness was related to higher levels of both cognitive and affective empathy. This relationship was mediated by alexithymia, which had an inverse relationship with both interoceptive awareness and empathy. Based on our results it appears that mere sensitivity to one's heartbeat does not, or is not sufficient, to explain 
individual differences in the understanding of one's own emotions or differences in empathy. Our study suggests that cognitive processes contributing to the subjective experience of bodily feelings are needed to provide that link, which is supportive of Bird and Viding's SOME and the role of its 'affective representation system' (Bird \& Viding, 2014).

We found that participants with high functioning ASD had lower interoceptive sensitivity and lower interoceptive awareness than participants without ASD. However, it has proved difficult to unequivocally establish whether the relationship between interoceptive awareness and alexithymia is unique to autism or also occurs in the typical population, because our control sample lacked individuals with high alexithymia. Despite this, this research contributes to the growing evidence that within the ASD population there is a large subgroup of individuals with high levels of alexithymia (Hill et al., 2004) and low levels of interoceptive awareness (Shah et al., 2016a; Shah et al., 2016b). These individuals have lower levels of cognitive and affective empathy than the typical population and individuals with ASD without alexithymia. Further research is needed to investigate whether being alexithymic as well as autistic could have an additional impact on other aspects of social functioning, emotion regulation, and mental health problems such as anxiety and depression. The present and related recent findings may therefore impact on ASD diagnosis since they show the potential importance of distinguishing between symptoms and characteristics that are linked to ASD and those that are linked to alexithymia. Finally, a focus on interoceptive training and in particular, promoting a beneficial (mindful) attentional style towards bodily sensations may be fruitful avenues for future therapeutic approaches.

\section{$\underline{\text { References }}$}

Ainley, V., Maister, L., \& Tsakiris, M. (2015). Heartfelt empathy? No association between interoceptive awareness, questionnaire measures of empathy, reading the mind in the eyes task 
or the director task. Frontiers in Psychology, 6, 554

Bachevalier, J., \& Loveland, K. A. (2006). The orbitofrontal-amygdala circuit and selfregulation of social-emotional behavior in autism. Neuroscience \& Biobehavioral Reviews, 30(1), 97-117.

Bagby, R. M., Parker, J. D., \& Taylor, G. J. (1994). The twenty-item toronto alexithymia Scale-I. item selection and cross-validation of the factor structure. Journal of Psychosomatic Research, 38(1), 23-32.

Baron-Cohen, S., Leslie, A. M., \& Frith, U. (1985). Does the autistic child have a "theory of mind"? Cognition, 21(1), 37-46.

Baron-Cohen, S., \& Wheelwright, S. (2004). The empathy quotient: An investigation of adults with asperger syndrome or high functioning autism, and normal sex differences. Journal of Autism and Developmental Disorders, 34(2), 163-175.

Baron-Cohen, S., Wheelwright, S., Skinner, R., Martin, J., \& Clubley, E. (2001). The autismSpectrum quotient (AQ): Evidence from asperger syndrome/high-functioning autism, males and females, scientists and mathematicians. Journal of Autism and Developmental Disorders, 31(1), 5-17.

Ben-Sasson, A., Hen, L., Fluss, R., Cermak, S. A., Engel-Yeger, B., \& Gal, E. (2009). A metaanalysis of sensory modulation symptoms in individuals with autism spectrum disorders. Journal of Autism and Developmental Disorders, 39(1), 1-11.

Bird, G., \& Cook, R. (2013). Mixed emotions: The contribution of alexithymia to the emotional symptoms of autism. Translational Psychiatry, 3(7), e285.

Bird, G., Silani, G., Brindley, R., White, S., Frith, U., \& Singer, T. (2010). Empathic brain responses in insula are modulated by levels of alexithymia but not autism. Brain, 133, 1515-25.

Bird, G., \& Viding, E. (2014). The self to other model of empathy: Providing a new framework for understanding empathy impairments in psychopathy, autism and alexithymia. Neuroscience and Biobehavioral Reviews, 47, 520-532.

Blair, R. J. R. (2005). Responding to the emotions of others: Dissociating forms of empathy 
through the study of typical and psychiatric populations. Consciousness and Cognition, 14(4), 698-718.

Botvinick, M., Jha, A. P., Bylsma, L. M., Fabian, S. A., Solomon, P. E., \& Prkachin, K. M. (2005). Viewing facial expressions of pain engages cortical areas involved in the direct experience of pain. NeuroImage, 25(1), 312-319.

Brener, J., \& Ring, C. (2016). Towards a psychophysics of interoceptive processes: the measurement of heartbeat detection. Phil. Trans. R. Soc. B, 371(1708), 20160015.

Cameron, O. G. (2001). Interoception: The inside story; a model for psychosomatic processes. Psychosomatic Medicine, 63, 697-710.

Cook, R., Brewer, R., Shah, P., \& Bird, G. (2013). Alexithymia, not autism, predicts poor recognition of emotional facial expressions. Psychological Science, 24(5), 723-732.

Craig, A. D. (2002). How do you feel? interoception: The sense of the physiological condition of the body. Nature Reviews Neuroscience, 3(8), 655-666.

Craig, A. (2008). Interoception and emotion: A neuroanatomical perspective. Handbook of emotions (3rd ed., pp. 272-292) New York: Guildford Press.

Crane, L., Goddard, L., \& Pring, L. (2009). Sensory processing in adults with autism spectrum disorders. Autism, 13(3), 215-228.

Critchley, H. D. (2005). Neural mechanisms of autonomic, affective, and cognitive integration. Journal of Comparative Neurology, 493, 154-166.

Damasio, A. (2000). The feeling of what happens: Body and emotion in the making of consciousness Harvest Books.

Demurie, E., De Corel, M., \& Roeyers, H. (2011). Empathic accuracy in adolescents with autism spectrum disorders and adolescents with attention-deficit/hyperactivity disorder. Research in Autism Spectrum Disorders, 5(1), 126-134.

DuBois, D., Ameis, S. H., Lai, M., Casanova, M. F., \& Desarkar, P. (2016). Interoception in autism spectrum disorder: A review. International Journal of Developmental Neuroscience, 52, 104-111. 
Dunn, B. D., Stefanovitch, I., Evans, D., Oliver, C., Hawkins, A., \& Dalgleish, T. (2010). Can you feel the beat? Interoceptive awareness is an interactive function of anxiety-and depressionspecific symptom dimensions. Behaviour Research and Therapy, 48(11), 1133-1138.

Dziobek, I., Rogers, K., Fleck, S., Bahnemann, M., Heekeren, H. R., Wolf, O. T., \& Convit, A. (2008). Dissociation of cognitive and emotional empathy in adults with asperger syndrome using the multifaceted empathy test. Journal of Autism and Developmental Disorders, 38, 464-473. Eisenberg, N. (2000). Emotion, regulation and moral development. Annual Review of Psychology, 51, $665-697$

Elwin, M., Ek, L., Kjellin, L., \& Schröder, A. (2013). Too much or too little: Hyper-and hyporeactivity in high-functioning autism spectrum conditions. Journal of Intellectual and Developmental Disability, 38(3), 232-241.

Ernst, J., Northoff, G., Bäker, H., Seifritz, E., \& Grimm, S. (2012). Interoceptive awareness enhances neural activity during empathy. Human Brain Mapping, 34, 1615-1624.

Fan, Y. T., Chen, C., Chen, S. C., Decety, J., \& Cheng, Y. (2014). Empathic arousal and social understanding in individuals with autism: Evidence from fMRI and ERP measurements. Social Cognitive and Affective Neuroscience, 9(8), 1203-1213.

Fiene, L., \& Brownlow, C. (2015). Investigating interoception and body awareness in adults with and without autism spectrum disorder. Autism Research,

Fukushima, H., Terasawa, Y., \& Umeda, S. (2011). Association between interoception and empathy: Evidence from heartbeat-evoked brain potential. International Journal of Psychophysiology, 79(2), 259-265.

Garfinkel, S. N., Tiley, C., O'Keeffe, S., Harrison, N. A., Seth, A. K., \& Critchley, H. D. (2016). Discrepancies between dimensions of interoception in autism: Implications for emotions and anxiety. Biological Psychology, 114, 117-126.

Garfinkel, S. N., Seth, A. K., Barrett, A. B., Suzuki, K., \& Critchley, H. D. (2015). Knowing your own heart: Distinguishing interoceptive accuracy from interoceptive awareness. Biological Psychology, 104, 65-74. 
Garretson, H. B., Fein, D., \& Waterhouse, L. (1990). Sustained attention in children with autism. Journal of Autism and Developmental Disorders, 20(1), 101-114.

Gillberg, C. L. (1992). The emanuel miller memorial lecture 1991. Journal of Child Psychology and Psychiatry, 33(5), 813-842.

Golan, O., \& Baron-Cohen, S. (2006). Systemizing empathy: Teaching adults with asperger syndrome or high-functioning autism to recognize complex emotions using interactive multimedia. Development and Psychopathology, 18(02), 591-617.

Greimel, E., Schulte-Rüther, M., Kircher, T., Kamp-Becker, I., Remschmidt, H., Fink, G. R., . . . Konrad, K. (2010). Neural mechanisms of empathy in adolescents with autism spectrum disorder and their fathers. NeuroImage, 49(1), 1055-1065.

Grynberg, D., Chang, B., Corneille, O., Maurage, P., Vermeulen, N., Berthoz, S., \& Luminet, O. (2012). Alexithymia and the processing of emotional facial expressions (EFEs): Systematic review, unanswered questions and further perspectives. PLoS One, 7, e42429.

Grynberg, D., \& Pollatos, O. (2015). Perceiving one's body shapes empathy. Physiology \& Behavior, 140, 54-60.

Grynberg, D., Luminet, O., Corneille, O., Grèzes, J., \& Berthoz, S. (2010). Alexithymia in the interpersonal domain: A general deficit of empathy? Personality and Individual Differences, 49, 845-850.

Guttman, H., \& Laporte, L. (2002). Alexithymia, empathy, and psychological symptoms in a family context. Comprehensive Psychiatry, 43(6), 448-455.

Hayes, A. F. (2013). Introduction to mediation, moderation, and conditional process analysis: A regression-based approach. New York: Guilford Press.

Herbert, B. M., Herbert, C., \& Pollatos, O. (2011). On the relationship between interoceptive awareness and alexithymia: Is interoceptive awareness related to emotional awareness? Journal of Personality, 79, 1149-1175.

Herbert, B. M., Pollatos, O., \& Schandry, R. (2007). Interoceptive sensitivity and emotion processing: An EEG study. International Journal of Psychophysiology, 65, 214-227. 
Hill, E., Berthoz, S., \& Frith, U. (2004). Brief report: Cognitive processing of own emotions in individuals with autistic spectrum disorder and in their relatives. Journal of Autism and Developmental Disorders, 34(2), 229-235.

Jabbi, M., Bastiaansen, J., \& Keysers, C. (2008). A common anterior insula representation of disgust observation, experience and imagination shows divergent functional connectivity pathways. PLoS ONE, 3(8), e2939.

James, W. (1894). Discussion: The physical basis of emotion. Psychological Review, 1, 516-529 Kandasamy, N., Garfinkel, S. N., Page, L., Hardy, B., Critchley, H. D., Gurnell, M., \& Coates, J. M. (2016). Interoceptive ability predicts survival on a London trading floor. Scientific Reports, 6 , 32986.

Keysers, C., \& Gazzola, V. (2009). Expanding the mirror: Vicarious activity for actions, emotions, and sensations. Current Opinion in Neurobiology, 19(6), 666-671.

Khalsa, S. S., \& Lapidus, R. C. (2016). Can interoception improve the pragmatic search for biomarkers in psychiatry? Frontiers in Psychiatry, 7.

Khalsa, S. S., Rudrauf, D., Damasio, A. R., Davidson, R. J., Lutz, A., \& Tranel, D. (2008). Interoceptive awareness in experienced meditators. Psychophysiology, 45(4), 671-677.

Knapp-Kline, K., \& Kline, J. P. (2005). Heart rate, heart rate variability, and heartbeat detection with the method of constant stimuli: Slow and steady wins the race. Biological Psychology, 69(3), 387-396.

Knoll, J. F., \& Hodapp, V. (1992). A comparison between two methods for assessing heartbeat perception. Psychophysiology, 29(2), 218-222.

Linden, W., Wen, F., \& Paulhus, D. (1995). Measuring alexithymia: Reliability, validity, and prevalence. Advances in Personality Assessment, 10, 51-95.

Livingston, L. A., \& Livingston, L. M. (2016). Commentary: Alexithymia, not autism, is associated with impaired interoception. Frontiers in Psychology, 7.

Lord, C., Risi, S., Lambrecht, L., Cook Jr, E. H., Leventhal, B. L., DiLavore, P. C., . . Rutter, M. (2000). The autism diagnostic observation Schedule-Generic: A standard measure of social 
and communication deficits associated with the spectrum of autism. Journal of Autism and Developmental Disorders, 30(3), 205-223.

Marchesi, C., Brusamonti, E., \& Maggini, C. (2000). Are alexithymia, depression, and anxiety distinct constructs in affective disorders? Journal of Psychosomatic Research, 49, 43-49.

Meissner, K., \& Wittmann, M. (2011). Body signals, cardiac awareness, and the perception of time. Biological Psychology, 86, 289-297.

Mehling, W. (2016). Differentiating attention styles and regulatory aspects of self-reported interoceptive sensibility. Phil.Trans.R.Soc.B, 371(1708), 20160013.

Mehling, W. E., Price, C., Daubenmier, J. J., Acree, M., Bartmess, E., \& Stewart, A. (2012). The multidimensional assessment of interoceptive awareness (MAIA). PLoS ONE, 7, e48230.

Murphy, J., Brewer, R., Catmur, C., \& Bird, G. (2017). Interoception and psychopathology: a developmental neuroscience perspective. Developmental Cognitive Neuroscience, 23, 45-56. Nemiah, J., Freyberger, H., \& Sifneos, P. (1976). Alexithymia: A view of the psychosomatic process. Modern Trends in Psychosomatic Medicine, 3, 430-439.

Northoff, G., Heinzel, A., de Greck, M., Bermpohl, F., Dobrowolny, H., \& Panksepp, J. (2006). Self-referential processing in our brain-a meta-analysis of imaging studies on the self. NeuroImage, 31(1), 440-457.

Ochsner, K. N., Zaki, J., Hanelin, J., Ludlow, D. H., Knierim, K., Ramachandran, T., . . . Mackey, S. C. (2008). Your pain or mine? Common and distinct neural systems supporting the perception of pain in self and other. Social Cognitive and Affective Neuroscience, 3(2), 144-160. Parker, J. D., Taylor, G. J., \& Bagby, R. M. (2003). The 20-item toronto alexithymia scale: III. reliability and factorial validity in a community population. Journal of Psychosomatic Research, 55(3), 269-275.

Paulus, M. P., \& Stein, M. B. (2010). Interoception in anxiety and depression. Brain Structure and Function, 214(5-6), 451-463.

Phillips, G. C., Jones, G. E., Rieger, E. J., \& Snell, J. B. (1999). Effects of the presentation of false heart-rate feedback on the performance of two common heartbeat-detection tasks. 
Psychophysiology, 36(4), 504-510.

Porges, S. (1993). Body perception questionnaire. Laboratory of Developmental Assessment, University of Maryland,

Quattrocki, E., \& Friston, K. (2014). Autism, oxytocin and interoception. Neuroscience \& Biobehavioral Reviews, 47, 410-430.

Reniers, R. L., Corcoran, R., Drake, R., Shryane, N. M., \& Völlm, B. A. (2011). The QCAE: A questionnaire of cognitive and affective empathy. Journal of Personality Assessment, 93, 84-95. Rogers, K., Dziobek, I., Hassenstab, J., Wolf, O. T., \& Convit, A. (2007). Who cares? revisiting empathy in asperger syndrome. Journal of Autism and Developmental Disorders, 37, 709-715. Rueda, P., Fernández-Berrocal, P., \& Baron-Cohen, S. (2015). Dissociation between cognitive and affective empathy in youth with asperger syndrome. European Journal of Developmental Psychology, 12, 85-98.

Samson, A. C., Huber, O., \& Gross, J. J. (2012). Emotion regulation in asperger's syndrome and high-functioning autism. Emotion, 12(4), 659-666.

Schaefer, M., Egloff, B., \& Witthüft, M. (2012). Is interoceptive awareness really altered in somatoform disorders? testing competing theories with two paradigms of heartbeat perception. Journal of Abnormal Psychology, 121, 719-724.

Schandry, R. (1981). Heartbeat perception and emotional experience. Psychophysiology, 18, 483-488.

Schauder, K. B., Mash, L. E., Bryant, L. K., \& Cascio, C. J. (2014). Interoceptive ability and body awareness in ASD. Journal of Experimental Child Psychology, 131, 193-200.

Schulz, A., Lass-Hennemann, J., Sütterlin, S., Schächinger, H., \& Vögele, C. (2013). Cold pressor stress induces opposite effects on cardioceptive accuracy dependent on assessment paradigm. Biological Psychology, 93(1), 167-174.

Shah, P. (2016). Interoception: the eighth sensory system: Practical solutions for improving selfregulation, self-awareness and social understanding of individuals with autism spectrum and related disorders. Journal of Autism and Developmental Disorders, 46, 3193-3194. 
Shah, P., Catmur, C., \& Bird, G. (2016a). Emotional decision-making in autism spectrum disorder: the roles of interoception and alexithymia. Molecular Autism, 7, 43-52.

Shah, P., Hall, R., Catmur, C., \& Bird, G. (2016b). Alexithymia, not autism, is associated with impaired interoception. Cortex, 81, 215-220.

Silani, G., Bird, G., Brindley, R., Singer, T., Frith, C., \& Frith, U. (2008). Levels of emotional awareness and autism: An fMRI study. Social Neuroscience, 3(2), 97-112.

Singer, T., Seymour, B., O'Doherty, J., Kaube, H., Dolan, R. J., \& Frith, C. D. (2004). Empathy for pain involves the affective but not sensory components of pain. Science 303, 1157-1162.

Singer, T., Critchley, H. D., \& Preuschoff, K. (2009). A common role of insula in feelings, empathy and uncertainty. Trends in Cognitive Sciences, 13, 334-340.

Skokauskas, N., \& Gallagher, L. (2010). Psychosis, affective disorders and anxiety in autistic spectrum disorder: Prevalence and nosological considerations. Psychopathology, 43, 8-16. Terasawa, Y., Moriguchi, Y., Tochizawa, S., \& Umeda, S. (2014). Interoceptive sensitivity predicts sensitivity to the emotions of others. Cognition \& Emotion, 28, 1435-1448.

Whitehead, W. E., Drescher, V. M., Heiman, P., \& Blackwell, B. (1977). Relation of heart rate control to heartbeat perception. Biofeedback and Self-Regulation, 2(4), 371-392.

Wicker, B., Keysers, C., Plailly, J., Royet, J., Gallese, V., \& Rizzolatti, G. (2003). Both of us disgusted in my insula: The common neural basis of seeing and feeling disgust. Neuron, 40(3), 655-664.

Wiens, S., Mezzacappa, E. S., \& Katkin, E. S. (2000). Heartbeat detection and the experience of emotions. Cognition \& Emotion, 14(3), 417-427.

Zaki, J., Davis, J. I., \& Ochsner, K. N. (2012). Overlapping activity in anterior insula during interoception and emotional experience. NeuroImage, 62(1), 493-499. 